Wright State University

CORE Scholar

Kno.e.sis Publications

The Ohio Center of Excellence in Knowledge-

Enabled Computing (Kno.e.sis)

7-19-2019

\title{
IAMHAPPY: Towards An loT Knowledge-Based Cross-Domain Well-Being Recommendation System for Everyday Happiness
}

Amelia Gyrard

Wright State University - Main Campus, amelie.gyrard@wright.edu

Amit Sheth

Wright State University - Main Campus, amit@sc.edu

Follow this and additional works at: https://corescholar.libraries.wright.edu/knoesis

Part of the Bioinformatics Commons, Communication Technology and New Media Commons, Databases and Information Systems Commons, OS and Networks Commons, and the Science and Technology Studies Commons

\section{Repository Citation}

Gyrard, A., \& Sheth, A. (2019). IAMHAPPY: Towards An loT Knowledge-Based Cross-Domain Well-Being Recommendation System for Everyday Happiness. Smart Health, 15.

https://corescholar.libraries.wright.edu/knoesis/1160

This Article is brought to you for free and open access by the The Ohio Center of Excellence in Knowledge-Enabled Computing (Kno.e.sis) at CORE Scholar. It has been accepted for inclusion in Kno.e.sis Publications by an authorized administrator of CORE Scholar. For more information, please contact library-corescholar@wright.edu. 


\title{
IAMHAPPY: Towards An IoT Knowledge-Based Cross-Domain Well-Being Recommendation System for Everyday Happiness
}

\author{
Amelie Gyrard ${ }^{\mathrm{a}}$, Amit Sheth ${ }^{\mathrm{a}}$ \\ ${ }^{a}$ Ohio Center of Excellence in Knowledge-enabled Computing (Kno.e.sis), Wright State University, Ohio, USA
}

\begin{abstract}
Nowadays, healthy lifestyle, fitness, and diet habits have become central applications in our daily life. Positive psychology such as well-being and happiness is the ultimate dream of everyday people's feelings (even without being aware of it). Wearable devices are being increasingly employed to support well-being and fitness. Those devices produce physiological signals that are analyzed by machines to understand emotions and physical state. The Internet of Things (IoT) technology connects (wearable) devices to the Internet to easily access and process data, even using Web technologies (aka Web of Things).

We design IAMHAPPY, an innovative IoT-based well-being recommendation system to encourage every day people's happiness. The system helps people deal with day-to-day discomforts (e.g., minor symptoms such as headache, fever) by using home remedies and related alternative medicines (e.g., naturopathy, aromatherapy), activities to reduce stress, etc. To achieve this system, we build a web-based knowledge repository for emotion with a focus on happiness and well-being. The knowledge repository helps analyze data produced by IoT devices to understand users' emotions and health. The semantics-based knowledge repository is integrated with a rule-based engine to suggest recommendations to achieve everyday people's happiness. The naturopathy application scenario supports the recommendation system.
\end{abstract}

Keywords: Internet of Things (IoT), Recommender Systems (RS), Affective Science, Emotion, Happiness, Well-being, Wellness, Rule-based Reasoning, Inference Engine, Knowledge Directory Service, Semantic Ontology Interoperability, Ontology Validation, Reusability, Semantic Web of Things (SWoT), Semantic Web Technologies, Reusable Knowledge.

\section{Introduction}

Nowadays, well-being, fitness, healthy lifestyle, and diet habits have become central applications in our everyday life. For instance, Google Fit focuses on activity tracking (minutes, miles, calories, and steps). However, users do not necessarily understand calorie meaning. What does mean 2000 calories? What is the maximum amount of calories that we eat to maintain a healthy lifestyle per week or month? What does mean 10000 steps done today, is it good enough to stay healthy? Those Internet of Things (IoT)-based applications' weaknesses demonstrate the need for domain expertise to understand the meaning of visualized data [1].

Positive psychology 1 , such as well-being and happiness is the ultimate everyday people's goal [2] [3]. Well-being is the process of evaluating people in terms of being satisfied with their life [4]. According to the US National Wellness Institute: "Wellness is an active process through which people become aware of, and make choices towards a more successful existence." According to the World Happiness Report, happiness and well-being depend on health and economy: "Physical activities, personal behavior, nutrition, and lifestyle and encourage to prevent from diseases" [4]. Simon Sinek, a mentor for leadership, says: "Working hard for something that we do not care about is called

Email addresses: amelie@knoesis.org (Amelie Gyrard), amit@knoesis .org (Amit Sheth)

1 https://www.authentichappiness.sas.upenn.edu/ 
stress, working hard for something we love is call passion." The same situation produces two different emotional states: stress (negative feeling) or love (positive feeling). Research to support happiness at work [5] [6] is more and more investigated; job positions such as "happiness manager" or "Chief Happiness Officer'2 are emerging in companies. Negative diagnostics perceived by people such as anxiety, stress, and depression can be reduced by focusing on the positive emotional states: How to encourage people's everyday well-being and happiness?

Increasingly, Recommendation Systems (RS) are invading our lives (e.g., YouTube for videos, Amazon for products, and Netflix for movies). Existing RS system surveys do no address RS for happiness yet [7] (machine learning-based RS published in 2018) [8] [9] [10]. well-being RS (published in 2019) [4] illustrates the emerging research need. Investigating well-being applications for a healthy and happy lifestyle is time-consuming for users and requires an eagerness to learn. A research study correlates happiness and a healthy lifestyle [11]. Mental health applications using the IoT devices are surveyed in [12]. Those studies demonstrate the need for an IoT-based well-being recommendation system to encourage everyday happiness.

We designed the following Research Questions (RQ):

- RQ1: Which physiological data generated by sensors are needed to deduce users' emotions? (e.g., a stress sensor, the serotonin level helps understanding depression).

- RQ2: How to integrate knowledge from complementary domains? (e.g., naturopathy with mindful activities).

- RQ3: How to deduce meaningful information (e.g., data analytics) from sensor data? How to design the wellbeing recommendation system?

Contributions (C): We design IAMHAPPY, an IoT-based well-being recommendation system to encourage everyday people's happiness. A knowledge repository is necessary to understand well-being and happiness and build this recommendation system. The knowledge repository aggregates structured knowledge already designed by domain experts and published within scientific publications. The knowledge repository provides:

- C1: An IoT dictionary to unify sensor data to deduce emotional states to address RQ1 (explained in Section 3.2 .

- C2: Reusing ontology-based projects to address RQ2 and designing the ontology catalog for emotions, food, obesity, depression, fitness, and sleep (explained in Section 3.3).

- C3: A knowledge-based cross-domain recommendation system to address RQ3, supported by the "Semantic Interoperability for the Web of Things" white paper [13] (explained in Section 3.4].

Structure of the Paper: Section 2 reviews the related work. Section 3 explains our well-being cross-domain recommendation system which integrates structured knowledge. Section 4 mentions limitations and future extensions. Section 5 concludes the paper and provides future work.

\section{Related Work}

Well-being RS are introduced in Section 2.1. Internet of Things (IoT)-based applications to deduce emotions are mentioned in Section 2.2. Section 2.3 concludes this section, and the literature review is summarized in Table 6 .

\subsection{Well-Being Recommendation Systems}

We summarize existing well-being recommendation systems (also classified in Table 6). However, RS for advising people's happiness are still lacking. Smart Recommender System of Hybrid Learning (SRHL) [4] suggests healthy food for personalized well-being; and aims to prevent diseases. The hybrid RS (content-based and collaborative filtering), uses unsupervised machine learning algorithms. The recommendations take into account time, activity, location, monetary costs, ingredients, health, nutritional value, availability, and the effects of combining the ingredients. MiningMinds [14] design personalized well-being and health-care support system; it includes a rule-based system using context (location, activity, weather, and emotion). The system provides 40 contextual scenarios and is evaluated with

2 http: //bit. ly/2LaLbdd 
forty users (thirty males, ten females in the middle-aged group: 25-49 years, ten different nationalities) and ten domain experts. I-Wellness [15] is a RS for personalized wellness therapy that uses hybrid Case-Based Reasoning (CBR). The RS aims to be integrated within wellness websites to help users search for suitable personalized therapy treatment based on their health condition. The I-Wellness online system arranges flexible appointments for patients with a wellness center. I-Wellness comprises six modules: (1) User wellness information, (2) wellness recommendation, (3) package selection, (4) appointment scheduling, (5) point allocation, and (6) wellness monitoring. The prototype is not accessible online to be tested. Motivate [16] is a personalized context-aware RS Android smartphone application that promotes a healthy and active lifestyle. The application recommends twenty types of activities according to some constraints: location, agenda, weather, profile (e.g., can cycle), and time. The evaluation study is from 15 November to 25 December 2010 (5 weeks). Participants are 6 Android phone users (five male, one female) that are colleagues or friends, and worked five days a week. The average age of the participants was 37 years (range: 24-63 years). 5 of the participants have a healthy weight Body mass index (BMI), and 1 of them is slightly overweight.

\subsection{IoT-based Emotion Applications}

We classified IoT-based emotion applications as follows: positive psychology which refers to well-being, and negative psychology such as stress, anxiety, sleep disorders, and depression.

Positive psychology (e.g., well-being): MoodScope [17] is a mobile application which infers the user's mood using smartphone data and asking the user's emotional state to correlate mood and phone usage. The application addresses the following research question: Can a smartphone infer its user's mood with the information it already has? The application is evaluated with 32 participants over two months. BeWell is an Android smartphone application to monitor, model, and promote well-being [18]. The application track activities that impact physical, social, and mental well-being (e.g., sleep, physical activity, and social interactions) and provides feedback to promote better health. However, it does not address diet or stress. The same authors surveyed mobile phone sensing [19] and highlight the resource-sensitive reasoning challenge. Rabbi et al. [20] monitor well-being (which includes measures of depressive symptoms) by developing a mobile-sensing system. MobiMood is a mobile social application that enables groups of friends to share their moods [21] which concludes that positive emotions are shared more easily than negative emotions. Amendola et al. [22] use IoT devices for health monitoring and recognize daily action patterns (e.g., cooking, eating, bathing, taking medicine, and sleeping). Three scenarios have been considered: (1) tracking human motion inside rooms, (2) gesture recognition, and (3) remote monitoring and control of the overnight living environment.

Negative psychology (e.g., stress, anxiety, depression): Mental Health Monitoring Systems (NHMS) using IoT devices are surveyed in [12]; 23 publications have been classified according to the study type (e.g., bipolar disorder detection, migraine forecasting, depression detection, anxiety detection, stress detection, social phobia association, bipolar disorder association, anxiety association, and depression association. Various devices have been employed in those studies (e.g., eye sensor, heart rate variability, electrodermal activity, wrist accelerometer, galvanic skin response, skin conductance, spo2, photoplethysmogram, accelerometer, gyroscope, pressure sensitive, video cameras, VR headset, pupil-corneal reflection and head tracker, SMS, calls, screen, GPS, location, touchscreens, audio, contacts, videos, sound, head-mounted display, questionnaires).

Depression disorders. Kim et al. [23] analyze depression severity (normal, mild, severe) on 20 seniors (age between 69 and 90) during 90 days using Ambient Assisted Living technologies and classification models (e.g., Artificial Neural Network, Decision Tree, Bayesian Network, and Support Vector Machine).

Stress disorders. Stress detection using smartphones' accelerometer data and the k-means algorithm is explained in [24]. StressSense recognizes stress from human voice using smartphones [25]. AMMON (Affective and Mental health MONitor) is a mental health monitor application that analyzes human voice (Chang et al. 2011 [26]). A stress detector can help people manage their stress to deal with their depression.

Sleep disorders. Five projects are analyzing sleep disorders: 1) Ontology-driven data integration for clinical sleep research [27], 2) human sleep data exploratory analysis [28], 3) diagnosis of sleep apnea with ML using the Weka software [29], 4) obtrusive Sleep Apnea (OSA) using IoT [30], and 5) correlation between sleep and affective disorders, hypertension, heart disease, and diabetes [31].

\subsection{Shortcomings of the Literature Study}

We summarize the following limitations of the literature study: 
- Doing the systematic literature review is a time-consuming task. There is a need to share the literature review innovatively (e.g., a knowledge repository for emotions) to ease the work of future researchers.

- A lot of ontologies cannot be exploited since they are not accessible online. There is a need to disseminate best practices (e.g., following FAIR principles [32]). to ease the task of computers to analyze ontologies and extract meaningful domain knowledge automatically.

- There is a lack of prototypes/experiments (e.g., web service, web application) that can be easily reproduced or tested to understand the applications and illustrate the limitations clearly.

- There is no recommender system for happiness yet.

\section{IAMHAPPY: An IoT Knowledge-Based Cross-Domain Well-Being Recommendation System for Happiness}

Our well-being and happiness recommendation system architecture is presented in Section 3.1 The IoT dictionary for understanding users' emotions and sensor data is introduced in Section 3.2. Knowledge repositories to model emotion and related topics (e.g., food for the naturopathy scenario) are explained in Section 3.3 . The cross-domain recommendation systems are described in Section 3.4

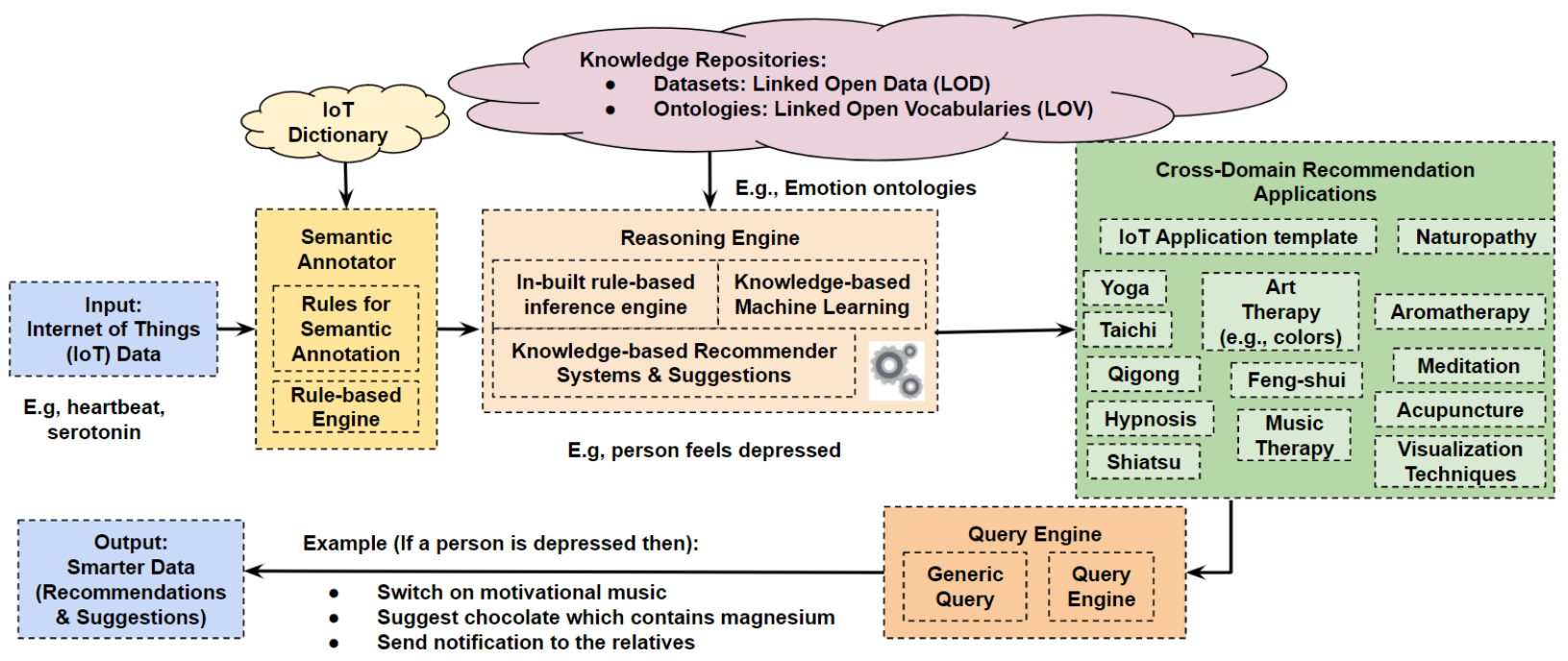

Figure 1: Overview architecture: semantic-based IoT cross-domain well-being recommendation system for happiness

\subsection{Architecture}

The architecture of the semantic-based IoT recommendation system for happiness is depicted in Figure 1 and comprises the following steps:

- IoT data (input): devices produce raw data (e.g., numbers). Data analysis is required to extract meaningful information from data.

- Semantic annotator unifies sensor data according to the IoT dictionary (explained in Section 3.2).

- Knowledge repositories model emotion and related topics for well-being and happiness (explained in Section 3.3 .

- Reasoning engine enriches IoT data with high-level information. The reasoning engine deduces the user's emotional states and needs (explained in Section 3.4.1). The current implementation uses a rule-based inference engine. As future work, knowledge-based machine learning techniques could be employed to deal with complex data (e.g., videos from cameras for face recognition, images).

- Recommender application provides user's suggestions based on domain-specific well-being applications for happiness and considering the user's emotional state and needs (explained in Section 3.4.2).

- Query engine retrieves a data subset that we are interested in designing specific applications. 
- Smart data (output): recommendations are returned to the users. The naturopathy recommendation application has been implemented as a first scenario (explained in Section 3.4.3.

\subsection{An IoT Dictionary for the Semantic Annotator}

We design an IoT dictionary ${ }^{3}$ which classifies devices that measure physiological signal sensor data to deduce users' moods and emotions. Table 6 references topics to address (e.g., stress) to design the IoT-based well-being recommendation system, and also references sensor measurement type for happiness and well-being to later process, unify, and enrich sensor data. This table enhances the classification of 18 sensor measurements in [12]. Hereafter, an example of sensors: 1) Hitachi's wearable sensor detects happiness based on physical movements. 2) Standford' sstress wearable device measuress cortisol in sweat. A stress monitoring patch senses skin conductance, skin temperature, and pulse wave [33]. 3) depression is measured when serotonin level is low (a neurotransmitter in the brain involved in regulating mood, appetite and sleep ${ }^{4}[34]$, which helps in understanding happiness. 4) Empatica E2 sensor $^{5}$ is a wrist-worn device which reads the skin conductivity, body temperature, and the body movement. The Empatica E4 wristband is a wearable research device that offers real-time physiological data acquisition and software for in-depth analysis and visualization, and 5) Affectiva $\mathbf{Q}$ sensor ${ }^{6}$ analyzes faces and speech to deduce emotion.

The IoT dictionary is displayed in our Graphical User Interface (GUI) accessible online $\mathrm{f}^{7}$ (also depicted in Figure 2 and Figure 3). Once a domain is chosen (e.g., healthcare or emotion), the list of sensors appear on the left (e.g., stress level). For each sensor, (1) a list of projects using this sensor can be retrieved ("Get Project" button), (2) a list of rules to interpret sensor data produced by this specific sensor can be retrieved ("Get Rule" button). The rules are compatible with the IoT dictionary (implemented as an ontology), and the rules follow the Jena rule syntax 8 and are executed with the Jena inference engine. Jena is an Apache open-source framework to develop semantic web applications. Rules are used for two different purposes: 1) the semantic annotation, and 2) the reasoning engine to infer high-level abstraction (e.g., high glucose level). The "Get Project" functionality retrieves projects referenced within the various catalogs referenced in Section 3.3 , and provides the veracity of our suggestions and keeps the provenance of the information (e.g., Figure 6 shows a tooltip citing a scientific publication).

\subsection{Knowledge Repositories for Emotion, Food, Obesity, Depression, Sleep, and Fitness}

To deduce meaningful information from IoT data produced by devices (mentioned above), we need common sense knowledge. We searched on Google and Google Scholar a set of specific keyphrases which 1) starts with ontology-based, 2) finishes with ontology, or 3) starts with semantic-based, knowledge-based, knowledge-graph or related synonyms. For instance, for the emotion domain, keyphrases are as follows: (1) Mood, positive mood, negative mood, (2) emotion, or specific emotions (anger, confusion, disgust, fear, happiness, sadness, shame, and surprise) - or synonym keyword, happiness (enjoy fun, eager), sadness (disliked, disappointed, bad and worst), (3) affective sciences, affective states, affective computing, (4) feeling, (5) well-being, etc.

This survey analysis is continuously enriched since several years (inspired from Agile software development methodologies) with new knowledge; we also take into considerations latest publications, surveys and we carefully analyze their reference sections that can introduce complementary topics and key scientific publications.

Most relevant scientific publications are referenced in Table 2, Table 3. Table 4, Table 5, and Table 6 when that information is provided:

- Ontology eases the reuse of the domain expertise already designed in previous projects. The column Ontology Availability (OA) explicitly describes if the ontology code is accessible online (URIs mentioned in the publication or after corresponding with the authors).

- Sensors used and measurement type to build our IoT dictionary (classified in Section 3.2).

$\sqrt[3]{\text { http: }: / / \text { sensormeasurement.appspot. } c o m / ? p=m 3}$

4 https://physicsworld.com/a/serotonin-sensor-diagnoses-depression/

5 http://www.empatica.com

6 http://www.affectiva.com/q-sensor/

7 http://linkedopenreasoning.appspot.com/?p=slorv2

8 https://jena.apache.org/documentation/inference/ 


\section{How to reason on sensor data?}

Select a domain and we provide you rules and projects for various sensors.

\section{Choose a domain}

Domain: Healthcare

\begin{tabular}{|c|c|c|}
\hline Sensor & Projects & \\
\hline $\begin{array}{l}\text { Pulse Oxymeter, SpO2, Blood Oxygen Saturation } \\
\text { Sensor Pulse oximetry is a method used to measure the } \\
\text { concentration of oxygen in the blood. }\end{array}$ & Get project & Get rule \\
\hline Stress Level Sensor Stress Level Sensor & Get project & Get rule \\
\hline $\begin{array}{l}\text { ECG or EKG (Electrocardiogram) ECG or EKG } \\
\text { (Electrocardiogram) }\end{array}$ & Get project & Get rule \\
\hline Blood Pressure Sensor & $\begin{array}{l}\text { Get project } \\
\text { Hypertens } \\
\text { m3:Diastol } \\
\text { - http://sen } \\
\text { NormalSy } \\
\text { greaterTha } \\
\text { m3:mmHg } \\
\text { - http://sen } \\
\text { NormalSy } \\
\text { greaterTha } \\
\text { NormalSys } \\
\text { - http://sen }\end{array}$ & $\begin{array}{l}\text { Get rule } \\
\text { ion - IF m3:SystolicBloodPressure greaterThan } 140 \text { m3:mmHg AND } \\
\text { icBloodPressure greaterThan } 90 \text { m3:mmHg THEN Hypertension } \\
\text { sormeasurement.appspot.com/RULES/LinkedOpenRulesHealth.txt } \\
\text { stolicBloodPressure Bravo - IF m3:SystolicBloodPressure } \\
\text { In } 120 \text { m3:mmHg AND m3:SystolicBloodPressure lessThan } 160 \\
\text { THEN NormalSystolicBloodPressure } \\
\text { sormeasurement.appspot.com/RULES/LinkedOpenRulesHealth.txt } \\
\text { stolicBloodPressure Hristoskova - IF m3:SystolicBloodPressure } \\
\text { in } 170 \text { m3:mmHg AND lessThan } 189 \text { m3:mmHg THEN } \\
\text { tolicBloodPressure } \\
\text { sormeasurement.appspot.com/RULES/LinkedOpenRulesHealth.txt }\end{array}$ \\
\hline
\end{tabular}

Figure 2: The health sensor taxonomy to retrieve rules to interpret health data

Select a domain and we provide you rules and projects for various sensors

Choose a domain

Domain: Emotion

Projects

Sensor

Stress Level Sensor Stress Level Sensor

ECG or EKG (Electrocardiogram) ECG or EKG

(Electrocardiogram)

SkinConductanceSensor, GSR (Galvanic Shin Response)
Get project Get rule

[Ko et al. 2006]. See LOV4IoT for more details. - Paper: Ontologybased context-aware service engine for U-Healthcare

\section{Get project Get rule}

[CodeBlue 2004]. See LOV4IoT for more details. - Paper: An adhoc sensor network infrastructure for emergency medical care. Ko et al. 2006]. See LOV4loT for more details. - Paper: OntologyU-Healthcare

Get project Get rule

[Ko et al. 2006]. See LOV4loT for more details. - Paper: Ontologybased context-aware service engine for U-Healthcare

Figure 3: The sensor taxonomy for Affective Science must be extended to retrieve existing projects and relevant rules to enrich data 
- The reasoning employed to analyze sensor data. Sometimes, rules can be reused to interpret data in other applications using the same sensors efficiently. Reasoning column within Table 2, Table 3 , Table 4, Table 5, and Table 6 reference the reasoning mechanism employed within the projects.

- Cited publications enriched our domain knowledge repository with scientific papers to prove the veracity of facts mentioned in this paper.

Our knowledge repository is the result of a continuous enrichment of the LOV4IoT knowledge repository [35] since 2012, an innovative solution to share Systematic Literature Review (SLR) (SLR guidelines [36]) as a tool rather than a survey paper. Ontologies enable us to share and reuse knowledge by designing concepts and relationships within a specific domain [37]. We intended knowledge catalogs to cover various topics (emotion, food, fitness, obesity, sleep, stress, and depression) relevant to well-being and happiness as described below.

Emotion knowledge catalog. We have collected and classified thirteen ontology-based emotion scientific projects (as referenced in Table 2), from 2005 to 2015: 1) ontologies that are open-source that define domain knowledge (column ontology availability with a green check mark), 2) ontology not openly accessible (column ontology availability with a red cross mark). We provide an open-source dataset and web service to easily retrieve the list of project, their ontology URLs, scientific publications, etc. Table 2 demonstrates that frequently, ontologies are not accessible online. The Semantic Web Community Best practice ${ }^{10}$ provide guidelines to ease the reuse of published ontologies.

Food knowledge catalog 11 . Similarly, we built the food knowledge base which collects a set of ontologies describing food, recipes, etc. It references about 36 ontology-based projects, as referenced in Table 3 and Table 4 . The food ontology dataset provides a subset with only 12 ontologies (Table 4) which shared their ontology code online for future automatic knowledge extraction (explanations provided in Section 4 ).

Fitness knowledge catalog ${ }^{12}$. In addition, knowledge related to fitness is collected. For instance, Villalonga et al. [38] encourage physical activities to prevent prevalent chronic health conditions, including cancer, cardiovascular diseases, obstructive lung illnesses, and diabetes, which are strongly connected to lifestyle. An ontology-based Motivational Messages for Physical Activity Coaching [38] (referenced in Table 5) classifies (1) Activities (sedentary, mild, vigorous), (2) indoor or outdoor locations, (3) actions are taking place in the different parts of the day.

Obesity knowledge catalog. The set of obesity ontologies are referenced in Table 5 , The obesity ontology (Sogic et al. [39]) describe the physical status, physical activity, and behavior, physiological status, and nutritional habits, of a teenage population. The 76 rules Semantic Web Rules Language (SWRL) deduce underweight condition, obese condition, overweight condition, Body Mass Index (BMI) or if in health. The rules are executed with the Pellet reasoner. Obesity management ontology (Kim et al. [40]) for Android mobile applications is developed in seven phases: (1) Defining the scope of obesity management, (2) selecting an ontology, (3) extracting the concepts, (4) assigning relationships between these concepts, (5) evaluating representative layers of ontology content, (6) representing the ontology formally with protege, and (7) developing a prototype application for obesity management. Other obesity ontologies are designed (Estanol et al. [41], Scala et al. [42]).

Additional projects helping understand disorders that can hinder happiness are referenced in Table 5 1) Depression knowledge catalog ${ }^{13}$ references a set of ontology-based depression projects [43] [44], DepressionKG [45], and mental health ontology [46], 2) sleep knowledge catalog references the sleep activity ontology to interpret data and understand sleep disorders [27], and 3) stress knowledge catalog references the human stress ontology [47].

\subsection{A Cross-Domain IoT-Based Well-Being Recommendation System for Happiness}

The data workflow is explained in Section 3.4.1 The cross-domain recommendation applications are introduced in Section 3.4.2 As an example, the naturopathy application is detailed in Section 3.4.3. The rule dataset evaluation is introduced in Section 3.4.4

$\sqrt[s]{\text { http: //lov4iot.appspot.com/?p=lov4iot-emotion }}$

http://lov4iot.appspot.com/?p=queryEmotionOntologiesWS

ichttp://iswc2018.semanticweb.org/call-for-resources-track-papers/

${ }_{11}$ http://lov4iot.appspot.com/?p=lov4iot-food.

http://lov4iot.appspot.com/?p=queryFoodontologiesWS

${ }^{12}$ https://lov4iot. appspot. com/?p=ontologies (see "Fitness, Sport Ontology Catalog" section.

Similarly, for the following knowledge catalogs, look for a specific keyword.)

13 https://lov4iot.appspot.com/?p=lov4iot-depression 


\begin{tabular}{|l|l|}
\hline Steps & Description \\
\hline Step 1 & $\begin{array}{l}\text { The raw measurements generated by the sensors are transformed into metadata with additional attributes: } \\
\text { (1) Unit of Measurement, (2) Timestamp, (3) Software Version, (4) Name, (5) Type, and (6) Domain of Operation. }\end{array}$ \\
\hline Step 2 & $\begin{array}{l}\text { The framework encodes the metadata using Sensor Markup Language } \\
\text { before converting into RDF to enable semantic reasoning. }\end{array}$ \\
\hline Step 3 & $\begin{array}{l}\text { Semantic reasoning drives higher level abstractions as new domain concepts. In the health domain, } \\
\text { the reasoning engine explicitly deduces the "flu" concept; in the weather domain, the "hot" concept. }\end{array}$ \\
\hline Step 4 & $\begin{array}{l}\text { The respective domain ontologies are used to classify these new concepts; } \\
\text { "flu" as a disease and "hot" as a seasonal condition. }\end{array}$ \\
\hline Step 5 & The respective domain datasets are used to link data (e.g., food with diseases, menu with season). \\
\hline Step 6 & $\begin{array}{l}\text { The concepts, rules, and datasets of the two domains, are combined } \\
\text { and cross-domain semantic reasoning takes place. In this example, the cross-domain reasoning } \\
\text { produces suggestions for recipes appropriate for a given state of health and the prevailing weather conditions. } \\
\text { The recommendations can be acted upon both by end-users and intelligent machines. }\end{array}$ \\
\hline
\end{tabular}

Table 1: Step descriptions of the generic IoT knowledge-based cross-domain recommendation engine [13] [48] [49]

\subsubsection{A Data Workflow to Enrich IoT Data}

The data workflow (depicted in Figure 4 and Figure 5 ) and the naturopathy scenario have been already taken as a baseline within the "Semantic Interoperability for the Web of Things" white paper [13], where authors are from different IoT standardization activities (W3C Web of Things, oneM2M, IEEE P2413, and AIOTI) and companies. In Figure 4 each step is detailed in Table 1 Step 2 uses the IoT dictionary explained in Section 3.2. Step 3 executes the rules. Step 4 and step 5 aggregates domain knowledge introduced in Section 3.3

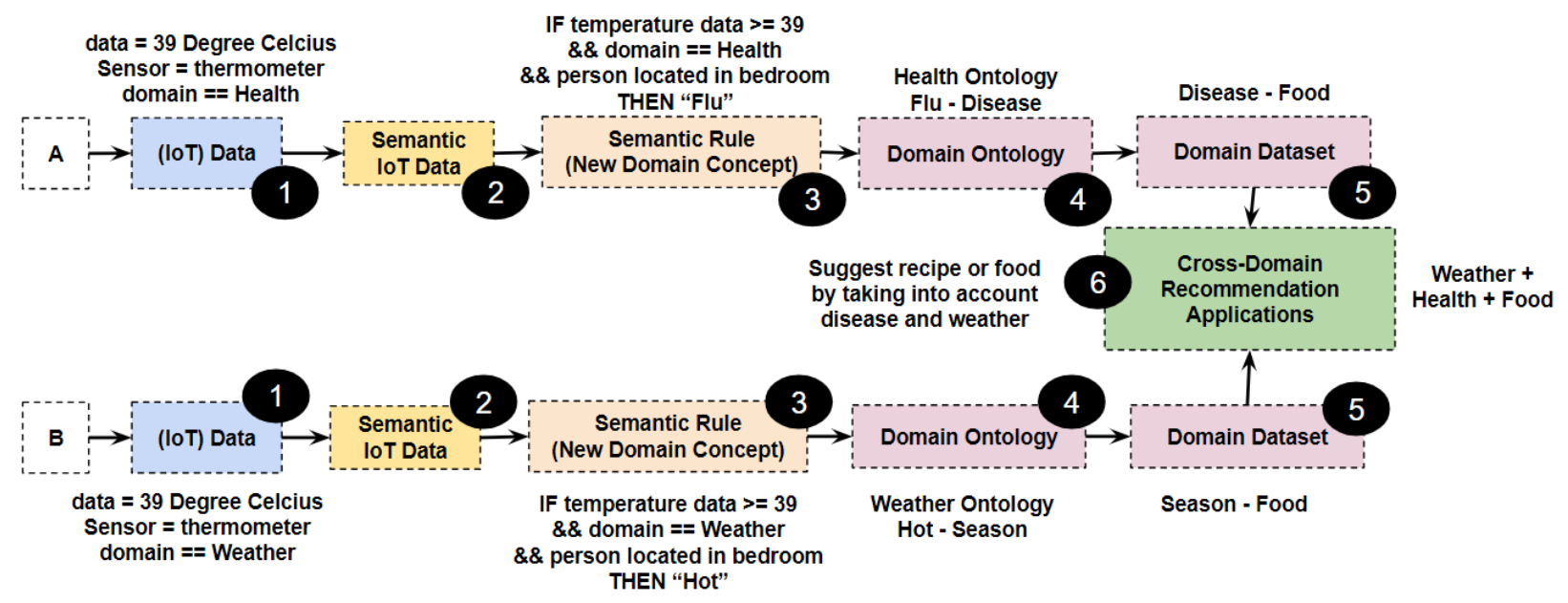

Figure 4: The generic IoT knowledge-based cross-domain recommendation engine [13] [48] [49]

Figures 2 and Figure 3 demonstrate the usage of the sensor taxonomy (previously explained in Section 3.2. The health IoT dictionary prototype retrieves ontology-based projects which help deduce meaningful information from health IoT data (see Figure 2). This reasoner can be enriched and extended for the emotion domain with a focus on happiness and well-being (see Figure 3 ). The reasoning engine prototype for healthcare is accessible online ${ }^{14}$

\subsubsection{Cross-Domain Recommendation Applications}

The recommendation system is reliable since it provides suggestions based on scientific publications in the following cross-domains:

\footnotetext{
${ }^{14}$ See footnote 7
} 
- Meditative physical activity (e.g., tai chi). Tai Chi helps with depression, pulmonary disease, balance disorders, Parkinson's disease, cardiovascular health, osteoporosis, chronic pain, and cancer as it is scientifically proven in [50].

- Breathing exercises (e.g., qigong) improves well-being and reduces anxiety, stress, and depression [51].

- Mindfulness activities (e.g., meditation, hypnosis) by listening to audios or watching videos. The MindAmend company $\sqrt{15}$ provides isochronic tones tracks for meditation, focus, energy, sleep, stress, tiredness, and high blood pressure.

- Music therapy helps a person to calm down his brain waves and can reduce stress (which can lead to depression) [52]. Internet of Musical Things is an emerging topic [53].

- Art therapy uses the usage of colors. Relationships between emotion and color are explained by Nijdam [54] and Wexner [55] (e.g., the angry mood is associated with a red color, blue-sad, yellow-happy). Mandala art therapy for adults is increasingly used for anxiety reduction [56].

- Healthy environment (e.g., feng-shui) improves the well-being and energy within a building [57].

- Visualization techniques [58] (e.g., successful people such as Olympic athletes employ this method [59]). It is also used to reduce stress, brings joy into your life, etc.

- Well-being activities (e.g., yoga) improves the quality of life and helps in dealing with chronic illness [60] (Chapter 7 focused on Yoga).

- Naturopathy: Natural products, such as herbs, prebiotic, probiotics, and selective medical diets, help for a healthy lifestyle [61]. The "Clinical naturopathy: an evidence-based guide to practice" book [61] addresses numerous diseases and syndromes (e.g., food allergy/intolerance, asthma, hypertension, stroke, anxiety, depression, insomnia). Correlations between naturopathy and yoga therapy are demonstrated in [62], supported by the Central Council for Research in Yoga and Naturopathy, and the State Ayurvedic College and Hospital, India. Additional fields, such as aromatherapy are highly relevant.

- Alternative medicines: Mind and body practices including acupuncture, manual therapies (e.g., spinal manipulation/mobilization), shiatsu, etc. Acupuncture ontologies have been included within Table 5 For instance, an ontology to describe meridians combined with modern medicine using the decision tree algorithm to detect psychiatric disorders is designed by Lin [63].

\subsubsection{The Naturopathy Application}

We propose innovative complementary and integrative health approaches such as the naturopathy recommendation application using knowledge-based techniques and based on scientific publications from domain experts.

Several naturopathy scenarios are already implemented to demonstrate the feasibility of the recommendation system, that is accessible online ${ }^{16}$ In all recommendation applications, suggestions are considered as reliable since they come from scientific publications that are mentioned above and within the demonstrators. As an example, we have the naturopathy application, which provides several scenarios (as discussed in Section 3.4.3):

- Scenario 1: suggesting home remedies according to the body temperature depicted in Figure 6 This scenario recommends home remedies when the temperature is too high; the reasoning engine infers the person might have the fever. The tooltip provides scientific publications to give the veracity of the recommendation.

- Scenario 2: Suggesting food according to the outside temperature.

- Scenario 3: Deducing mood according to the external luminosity.

- Scenario 4: Deducing mood or diseases from heartbeat, skin conductance, and blood pressure.

- Scenario 5: Suggesting a recipe according to the food available in your kitchen.

A detailed description of the naturopathy scenario can be found in [48] [49]. Additional scenarios can be easily integrated into our system.

\subsubsection{Evaluation of the Rule Dataset}

We evaluate the rule dataset with two metrics: 1) correctness that means that are no incompatibility with other rules, and 2) completeness that means that high-level information covers all sensor values. When integrating a new rule

\footnotetext{
15 https: / / www.mindamend.com/

16 http://sensormeasurement.appspot. com/?p=naturopathy
} 


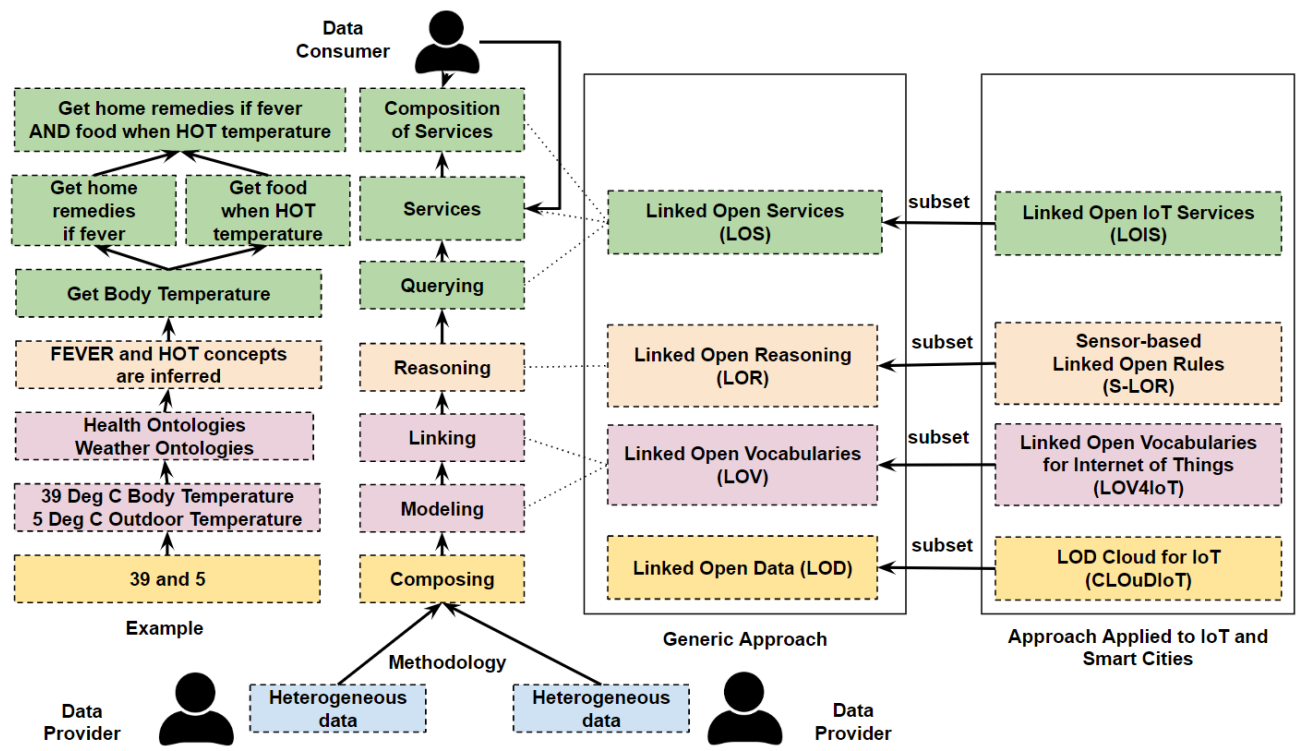

Figure 5: Using the Web of Knowledge and IoT applications to build the naturopathy scenario 64

\section{Suggesting home remedies according to body temperature}

1. This scenario is based on: M3 RDF health data

2. M2M Aggregation Gateway (Convert Health Measurements into Semantic Data): Convert health measurements

3. We deduce that the temperature corresponds to the body temperature.

4. We deduce that the person is sick.

5. We propose all fruits/vegetables according to this disease.

6. M2M Application: Temperature $=>$ Cold $=>$ Food: (Wait 10 seconds!) Food if you are sick

Scientific publications are provided to prove the veracity

of the recommendations.

- Name=temperature, Value $=38.7$, Unit $=\mathrm{Cel}$, InferType $=$ Body Temperature, Deduce $=$ HighFever, Suggest $=$ Pepper mint

- Name=temperature, Value $=38.7$, Unit $=\mathrm{Cel}$, InferType $=$ Body Temperature, Deduce $=$ HighFever, Suggest $=$ Thyme

- Name=temperature, Value $=38.7$, Unit $=C$ Cel, InferType $=$ Body Temperature, Deduce $=$ HighFever. Suggest $=$ Cinnamon

- Name=temperature, Value $=38.7$, Unit=Cel, InferType $=$ Body Temperatu See paper: Honey as complementary medecine: A review [Singh et al. 2012]

- Name=temperature, Value $=38.7$, Unit=Cel, InferType = Body Temperat lemon, clover, milk, cinnamon and water for treatments of various ailments a

- Name=temperature, Value $=38.7$, Unit=Cel, InferType $=$ Body Temperature, Deduce $=$ HighFever, Suggest $=$ Lemon

Figure 6: Naturopathy Scenario 1: Suggesting home remedies according to the body temperature 
within the rule dataset, correctness must remain. The documentation ${ }^{17}$ provides tables that summarize completeness and correctness are ensured per sensor type.

\section{Limitations and Future Extensions}

We introduce the limitations that we are aware of and future extensions to consider.

\subsection{Planned Evaluation}

The first evaluation phase of our IAMHAPPY recommendation application will be with users (e.g., researchers). Ideally, a second evaluation phase would be with depressed-diagnosed patients to demonstrate they become happier by using guidelines to evaluate people's happiness. Physiological datasets can be used to assess our computer-system approach.

User's feedback. Ideally, our recommendation system will be evaluated with user's feedback (e.g., 25 researcher colleagues) according to usability and usefulness criteria to determine the feasibility of the system. It helps to refine the RS application. Then, the improved RS can be tested with patients. User population considered is mainly sedentary lifestyles as opposed to construction workers (since they already practice physical activities). To achieve this, we need front-end developers to design an ergonomic and user-friendly application.

Guidelines to evaluate the patient's happiness and reduce depression. A robust evaluation requires patients diagnosed with depression; a partnership with psychological clinicians would be ideal. The RS application evaluation is considered as successful if the patients are happier by using every day the application. Patient's happiness can be measured with those guidelines: 1) The Satisfaction With Life Scale (SWLS) [65] is a guideline to assess the overall assessment of life satisfaction. 2) The Oxford Happiness Questionnaire (OHQ) measures personal happiness [66]; it comprises a set of 29 multiple-choice well-being and happiness items. 3) The Gratitude Questionnaire (GQ-6) guideline regulates the gratitude, which increases happiness and reduces depression according to Robert Emmons, a leading gratitude researcher, also recognized for positive psychology. 4) The Circumplex mood model [67] employs dimensions (pleasure and activeness) to describe and measure mood. The pleasure dimension measures how positive or negative someone feels. The activeness dimension measures whether someone takes an active or passive action under the mood state. 5) The Oldenburg burnout inventory [67].

Datasets. Before deploying IoT devices, datasets providing physiological signals can be used to assess our recommender application: 1) The DEAP datase 18 comprises 32 patients, and is designed for emotion analysis using physiological signals such as GSR, blood volume pressure, respiration pattern, skin temperature, EMG, EOG, and EEG [68]. 2) The heart rate datase ${ }^{19}$ comprises 270 patients' data (age, sex, chest pain type, resting blood pressure, serum cholesterol, fasting blood sugar, resting electrocardiographic results, maximum heart rate achieved, etc.) [69]. 3) The ECG datase ${ }^{20}[70]$. 4) The PhysioBank datase ${ }^{21}$ 5) The World Happiness Report dataset on Kaggle ${ }^{22}$ surveys the state of global happiness in countries. 6) Google Dataset Search ${ }^{23}$ or the Linked Open Data Cloud $24[71]$ are dataset repositories.

\subsection{Technical Limitations}

The cold-start problem is a common issue when designing recommender systems. Hybrid recommender systems: content-based filtering systems personalize the application according to user's needs, and collaborative filtering systems are based on users' appreciations, help overcome the cold-start problem. Our first iteration of recommendations will be a ground-truth based on scientific publications mentioned in Section 2. For instance, within the naturopathy scenario, we suggest honey as a home remedy by citing a scientific publication [72].

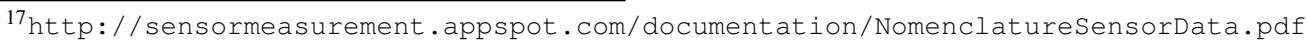

18 http://www.eecs.qmul.ac.uk/mmv/datasets/deap/

19 https://archive.ics.uci.edu/ml/datasets/Statlog+(Heart)

20 http://www.cs.ucr.edu/ eamonn/time_series_data/

${ }^{21}$ http://physionet.org/physiobank/database/

22 https://www.kaggle.com/mrisdal/happiness-and-open-data

23 https://toolbox.google.com/datasetsearch

24 https://lod-cloud.net/\#
} 
The automatic statistical analysis of knowledge is a parallel work to consider to study a set of IoT ontologies automatically (this research leads to an outstanding paper award in 2018 [73]). An extension refined the research for smart home, weather, and smart city topics [74]. A similar approach can be applied to the topics mentioned in this work: (1) fitness/activity, obesity, sleep, stess, depression ontologies (Table 5), (2) affective science and Emotion (Table 2), and (3) food ontologies (Table 3 and Table 4).

\section{Conclusion and Future Work}

We designed an IoT-based well-being recommendation system to advise people to feel happier, which is the ultimate goal of everyday people's feelings. The naturopathy recommendation application supports the recommendation system. Our innovative cross-domain recommendation system classifies, and analyzes the common sense knowledge (e.g., emotion, food, fitness, obesity, sleep, stress, and depression), released as ontology catalogs, required to understand happiness to build well-being applications. Those knowledge catalogs support researchers with the Systematic Literature Survey, which is a time-consuming task and requires an eagerness to learn and investigate existing projects. The knowledge catalogs encourage researchers to follow FAIR principles and share their reproducible experiments by publishing online their ontologies, datasets, rules, etc.

\section{References}

[1] A. Sheth, Internet of Things to Smart IoT Through Semantic, Cognitive, and Perceptual Computing, IEEE Intelligent Systems.

[2] M. Seligman, Flourish: A Visionary New Understanding of Happiness and Well-Being (Book), Simon and Schuster, 2012.

[3] D. Lama, The Art of Happiness: A Handbook for Living (Book). The author got the Nobel Peace Price in 1989, Penguin, 2009.

[4] R. Nouh, et al., A Smart Recommender Based on Hybrid Learning Methods for Personal Well-Being Services, MDPI Sensors Journal (IF: 3.031 in 2018).

[5] G. Van Gils, Happier at Work: The Power of Love to Transform the Workplace, She Writes Press, 2017.

[6] A. McKee, How to Be Happy at Work: The Power of Purpose, Hope, and Friendship, Harvard Business Press, 2017.

[7] I. Portugal, et al., The Use of Machine Learning Algorithms in Recommender Systems: a Systematic Review, Elsevier Expert Systems with Applications Journal (IF: 3.768 in 2017).

[8] J. Lu, D. Wu, M. Mao, W. Wang, G. Zhang, Recommender System Application Developments: a Survey, Elsevier Decision Support Systems Journal (IF: 3.565 in 2017).

[9] J. Bobadilla, F. Ortega, A. Hernando, A. Gutiérrez, Recommender Systems Survey, Elsevier Knowledge-Based Systems Journal (IF: 4.396 in 2017).

[10] G. Adomavicius, A. Tuzhilin, Toward the Next Generation of Recommender Systems: A Survey of the State-of-the-Art and Possible Extensions, IEEE Transactions on Knowledge \& Data Engineering (IF: 2.775 in 2017).

[11] R. Veenhoven, Healthy Happiness: Effects of Happiness on Physical Health and the Consequences for Preventive Health Care, Springer Journal of Happiness Studies (IF: 1.986 in 2017).

[12] Mental Health Monitoring with Multimodal Sensing and Machine Learning: A survey, Elsevier Pervasive and Mobile Computing Journal (IF: 2.974 in 2017).

[13] P. Murdock, L. Bassbouss, M. Bauer, M. B. Alaya, R. Bhowmik, P. Brett, et al., Semantic Interoperability for the Web of Things (White Paper) (2016).

[14] M. Afzal, S. I. Ali, R. Ali, M. Hussain, T. Ali, W. A. Khan, M. B. Amin, B. H. Kang, S. Lee, Personalization of Wellness Recommendations using Contextual Interpretation, Elsevier Expert Systems with Applications Journal (IF: 3.768 in 2017).

[15] T. P. Lim, W. Husain, N. Zakaria, Recommender System for Personalized Wellness Therapy, International Journal of Advanced Computer Science and Applications (IF: 1.324 in 2017).

[16] Y. Lin, J. Jessurun, B. De Vries, H. Timmermans, Motivate: Towards Context-Aware Recommendation Mobile System for Healthy Living, in: International Conference on Pervasive Computing Technologies for Healthcare (PervasiveHealth) and Workshops, IEEE, 2011.

[17] R. LiKamWa, Y. Liu, N. D. Lane, L. Zhong, Can your Smartphone Infer your Mood?, in: PhoneSense Workshop, 2011.

[18] N. D. Lane, M. Mohammod, M. Lin, X. Yang, et al., Bewell: A Smartphone Application to Monitor, Model and Promote Wellbeing, in: International Conference on Pervasive Computing Technologies for Healthcare (Conference ranking not found), 2011.

[19] N. D. Lane, et al., A Survey of Mobile Phone Sensing, IEEE Communications Magazine (IF: 10.356 in 2018).

[20] M. Rabbi, S. Ali, T. Choudhury, E. Berke, Passive and In-Situ Assessment of Mental and Physical Well-Being Using Mobile Sensors, in: ACM International conference on Ubiquitous computing (UbiComp, A-Rank Conference), ACM, 2011.

[21] K. Church, E. Hoggan, N. Oliver, A study of Mobile Mood Awareness and Communication through MobiMood, in: Nordic Conference on Human-Computer Interaction: Extending Boundaries, ACM, 2010.

[22] S. Amendola, R. Lodato, S. Manzari, C. Occhiuzzi, G. Marrocco, RFID Technology for IoT-Based Personal Healthcare in Smart Spaces, IEEE Internet of Things Journal (IF: 5.863 in 2017).

[23] J.-Y. Kim, N. Liu, H.-X. Tan, C.-H. Chu, Unobtrusive Monitoring to Detect Depression for Elderly with Chronic Illnesses, IEEE Sensors Journal (IF: 2.617 in 2017).

[24] E. Garcia-Ceja, V. Osmani, O. Mayora, Automatic Stress Detection in Working Environments from Smartphones' Accelerometer Data: a First Step, IEEE Journal of Biomedical and Health Informatics (IF: 3.85 in 2017). 
[25] H. Lu, D. Frauendorfer, M. Rabbi, M. S. Mast, G. T. Chittaranjan, A. T. Campbell, D. Gatica-Perez, T. Choudhury, StressSense: Detecting Stress in Unconstrained Acoustic Environments Using Smartphones, in: ACM Conference on Ubiquitous Computing (UbiComp, A-Rank Conference), ACM, 2012.

[26] K.-h. Chang, D. Fisher, J. Canny, B. Hartmann, How's my Mood and Stress?: an Efficient Speech Analysis Library for Unobtrusive Monitoring on Mobile Phones, in: Body Area Networks Conference (Conference ranking not found), Institute for Computer Sciences, Social-Informatics, 2011.

[27] R. S. Mueller, Ontology-Driven Data Integration for Clinical Sleep Research, Ph.D. thesis, Case Western Reserve University (2011).

[28] P. Laxminarayan, Exploratory Analysis of Human Sleep Data, Ph.D. thesis, Worcester Polytechnic Institute (2004).

[29] R. Angelidou, Development of a Portable System for Collecting and Processing Bio-signals and Sounds to Support the Diagnosis of Sleep Apnea, Master's thesis (2015).

[30] D. C. Yacchirema, D. Sarabia-Jácome, C. E. Palau, M. Esteve, A Smart System for Sleep Monitoring by Integrating IoT with Big Data Analytics, IEEE Access Journal (IF: 3.557 in 2017).

[31] G. G. Alvarez, N. T. Ayas, The Impact of Daily Sleep Duration on Health: a Review of the Literature, Progress in cardiovascular nursing.

[32] M. D. Wilkinson, M. Dumontier, I. J. Aalbersberg, G. Appleton, M. Axton, A. Baak, N. Blomberg, J.-W. Boiten, L. B. da Silva Santos, P. E. Bourne, et al., The FAIR Guiding Principles for Scientific DataManagement and Stewardship, Nature Scientific Data Journal (IF: 5.305 in 2017).

[33] S. Yoon, J. K. Sim, Y.-H. Cho, A Flexible and Wearable Human Stress Monitoring Patch, Nature Scientific Reports Journal (IF: 4.122 in 2017).

[34] T. Vandenryt, B. van Grinsven, K. Eersels, P. Cornelis, S. Kholwadia, T. Cleij, R. Thoelen, W. De Ceuninck, M. Peeters, P. Wagner, SingleShot Detection of Neurotransmitters in Whole-Blood Samples by Means of the Heat-Transfer Method in Combination with Synthetic Receptors, MDPI Sensors Journal (IF: 3.031 in 2018).

[35] A. Gyrard, C. Bonnet, K. Boudaoud, M.Serrano, LOV4IoT: A Second Life for Ontology-Based Domain Knowledge to Build Semantic Web of Things Applications, in: IEEE International Conference on Future Internet of Things and Cloud, 2016.

[36] G. Rizzo, F. Tomassetti, A. Vetro, L. Ardito, M. Torchiano, M. Morisio, R. Troncy, Semantic Enrichment for Recommendation of Primary Studies in a Systematic Literature Review, Digital Scholarship in the Humanities.

[37] T. R. Gruber, Toward Principles for the Design of Ontologies Used for Knowledge Sharing?, International Journal of Human-Computer Studies.

[38] C. Villalonga, H. Hermens, L. J. Herrera, H. Pomares, I. Rojas, O. Valenzuela, O. Banos, et al., Ontological Modeling of Motivational Messages for Physical Activity Coaching, in: EAI International Conference on Pervasive Computing Technologies for Healthcare, ACM, 2017.

[39] A. Sojic, W. Terkaj, G. Contini, M. Sacco, Modularising ontology and designing inference patterns to personalise health condition assessment: the case of obesity, Journal of biomedical semantics.

[40] H.-Y. Kim, H.-A. Park, Y. H. Min, E. Jeon, Development of an Obesity Management Ontology-Based on the Nursing Process for the Mobile-Device Domain, Journal of Medical Internet Research (IF: 4.671 in 2017).

[41] M. Estañol, F. Masucci, A. Mosca, I. Ràfols, Mapping knowledge with ontologies: the case of obesity, Preprint arXiv:1712.03081.

[42] P. L. Scala, D. P. Di, D. Tresoldi, C. L. Lafortuna, G. Rizzo, M. Padula, Ontology-supported clinical profiling for the evaluation of obesity and related comorbidities., Studies in health technology and informatics.

[43] H. Jung, H.-A. Park, T.-M. Song, Ontology-based approach to social data sentiment analysis: detection of adolescent depression signals, Journal of Medical Internet Research (IF: 4.671 in 2017).

[44] Y.-S. Chang, W.-C. Hung, T.-Y. T. Juang, Depression Diagnosis Based on Ontologies and Bayesian Networks, Conference on Systems, Man, and Cybernetics.

[45] Z. Huang, J. Yang, F. van Harmelen, Q. Hu, Constructing Knowledge Graphs of Depression, in: Conference on Health Information Science, Springer, 2017.

[46] M. Hadzic, M. Chen, T. S. Dillon, Towards the Mental Health Ontology, in: Bioinformatics and Biomedicine Conference, IEEE, 2008.

[47] E. Nasiri Khoozani, M. Hadzic, Designing the human stress ontology: A formal framework to capture and represent knowledge about human stress, Australian Psychologist.

[48] A. Gyrard, C. Bonnet, K. Boudaoud, Enrich machine-to-machine data with semantic web technologies for cross-domain applications, in: World Forum on Internet of Things (WF-IOT), 2014.

[49] A. Gyrard, Designing Cross-Domain Semantic Web of Things Applications, Ph.D. thesis, Telecom ParisTech, Eurecom (2015).

[50] P. M. Wayne, M. Fuerst, The Harvard Medical School Guide to Tai Chi: 12 weeks to a Healthy Body, Strong Heart, and Sharp Mind, Shambhala Publications, 2013.

[51] F. Wang, J. K. Man, E.-K. O. Lee, T. Wu, H. Benson, G. L. Fricchione, W. Wang, A. Yeung, The Effects of Qigong on Anxiety, Depression, and Psychological Well-Being: a Systematic Review and Meta-Analysis, Evidence-Based Complementary and Alternative Medicine.

[52] Y. Pingle, IoT for Music Therapy, in: International Conference on Computing for Sustainable Global Development (Conference ranking not found), IEEE, 2016.

[53] D. Sang, Enhancing the Wellbeing of Older Adults Through IoT Design with Music, Master's thesis, NTNU (2018).

[54] N. Nijdam, Mapping Emotion to Color (2009).

[55] L. Wexner, The Degree to Which Colors (Hues) are Associated with Mood-Tones, Journal of Applied Psychology (IF: 4.643 in 2017).

[56] R. van der Vennet, S. Serice, Can Coloring Mandalas Reduce Anxiety? A Replication Study, Art therapy.

[57] M. Bonaiuto, E. Bilotta, A. Stolfa, Feng Shui and Environmental Psychology: A Critical Comparison, Journal of Architectural and planning research (H-Index: 22).

[58] S. Gawain, Creative Visualization: Use the Power of Your Imagination to Create What You Want in Your Life, New World Library, 2016.

[59] A. Guillot, Visualisation en Sports de Combat-Vaincre grace au Mental (Book in French), Amphora, 2012.

[60] T. G. Plante, Healing with Spiritual Practices: Proven Techniques for Disorders from Addictions and Anxiety to Cancer and Chronic Pain, ABC-CLIO, 2018. 
[61] J. Wardle, J. Sarris, Clinical Naturopathy: an Evidence-Based Guide to Practice, Elsevier Health Sciences, 2014.

[62] R. Rastogi, S. Rastogi, Concept and Role of Diet as a Component of Naturopathy and Yoga Therapy.

[63] Y. Lin, Design and Implementation of an Ontology-Based Psychiatric Disorder Detection System, WSEAS Transactions on Information Sciences and Applications (H-Index=17).

[64] A. Gyrard, P. Patel, A. Sheth, M. Serrano, Building the Web of Knowledge with Smart IoT Applications, IEEE Intelligent Systems Magazine.

[65] W. A. Arrindell, J. Heesink, J. A. Feij, The satisfaction with life scale (swls): Appraisal with 1700 healthy young adults in the netherlands, Personality and individual differences.

[66] P. Hills, M. Argyle, The Oxford Happiness Questionnaire: a Compact Scale for the Measurement of Psychological Well-Being, Personality and individual differences.

[67] E. Demerouti, A. B. Bakker, The Oldenburg Burnout Inventory: A Good Alternative to Measure Burnout and Engagement, Hauppauge, NY: Nova Science, 2008

[68] S. Koelstra, C. Muhl, M. Soleymani, J.-S. Lee, A. Yazdani, et al., DEAP: A Database for Emotion Analysis; using Physiological Signals, IEEE Transactions on Affective Computing (IF: 4.585 in 2017).

[69] F. Ahmed, An Internet of Things (IoT) Application for Predicting the Quantity of Future Heart Attack Patients, International Journal of Computer Applications (IF: 3.12 in 2017).

[70] F. Ganz, Intelligent Communication and Information Processing for Cyber-Physical Data, Ph.D. thesis, University of Surrey (2014).

[71] C. Bizer, T. Heath, T. Berners-Lee, Linked data-the story so far, International Journal on Semantic Web and Information Systems (IJSWIS).

[72] M. Sharma, D. Sharma, S. Khan, Honey as Complementary Medicine - A Review, International Journal of Pharma and Bio Sciences (IF: 7.291 in 2017).

[73] M. Noura, A. Gyrard, S. Heil, M. Gaedke, Concept Extraction from the Web of Things Knowledge Bases, in: International Conference WWW/Internet 2018, Elsevier, 2018, Outstanding Paper Award.

[74] M. Noura, A. Gyrard, S. Heil, M. Gaedke, Automatic Knowledge Extraction to Build Semantic Web of Things Applications.

[75] F. Berthelon, P. Sander, Emotion Ontology for Context Awareness, in: International Conference on Cognitive Infocommunications (CogInfoCom), IEEE, 2013.

[76] J. F. Sánchez-Rada, C. A. Iglesias, Onyx: A Linked Data approach to Emotion Representation, Information Processing \& Management.

[77] J. Hastings, B. Smith, W. Ceusters, M. Jensen, K. Mulligan, Representing mental functioning: Ontologies for mental health and disease, in: International Conference on Biomedical Ontology (ICBO), University of Graz, 2012.

[78] J. M. López, R. Gil, R. García, I. Cearreta, N. Garay, Towards an Ontology for Describing Emotions, in: World Summit on Knowledge Society, Springer, 2008

[79] M. Arguedas, F. Xhafa, T. Daradoumis, S. Caballe, An ontology about emotion awareness and affective feedback in elearning, in: International Conference on Intelligent Networking and Collaborative Systems (INCOS), IEEE, 2015.

[80] S. A. A. Tapia, A. H. F. Gómez, J. B. Corbacho, S. Ratté, J. Torres-Diaz, P. V. Torres-Carrion, J. M. Garcia, A contribution to the method of automatic identification of human emotions by using semantic structures, in: Conference on Interactive Collaborative Learning, IEEE, 2014.

[81] M. D. Sykora, T. Jackson, A. O’Brien, S. Elayan, Emotive ontology: Extracting fine-grained emotions from terse, informal messages.

[82] K. Fang, S.-C. Lin, Exploring the relationships between colors and main meridians: An ontology-based perspective, in: International Conference on Advanced Information Management and Service (IMS), 2010.

[83] M. Grassi, Developing HEO Human Emotions Ontology, in: European Workshop on Biometrics and Identity Management, Springer, 2009.

[84] F. Radulovic, N. Milikic, Smiley Ontology, in: Proceedings of The 1st International Workshop On Social Networks Interoperability, 2009.

[85] K.-I. Benta, A. Rarău, M. Cremene, Ontology Based Affective Context Representation, in: Euro American conference on Telematics and information systems, ACM, 2007.

[86] Z. Obrenovic, N. Garay, J. M. López, I. Fajardo, I. Cearreta, An Ontology for Description of Emotional Cues, in: International Conference on Affective Computing and Intelligent Interaction, Springer, 2005.

[87] Y. Y. Mathieu, Annotation of Emotions and Feelings in Texts, in: International Conference on Affective Computing and Intelligent Interaction, Springer, 2005

[88] S. Peroni, G. Lodi, L. Asprino, A. Gangemi, V. Presutti, FOOD: Food in Open Data, in: International Semantic Web Conference (A-rank conference), Springer, 2016.

[89] E. J. Griffiths, D. M. Dooley, P. L. Buttigieg, R. Hoehndorf, F. S. Brinkman, W. W. Hsiao, FoodON: A Global Farm-to-Fork Food Ontology. The Development of a Universal Food Vocabulary, in: ICBO/BioCreative, 2016.

[90] A. H. Celdran, F. J. G. Clemente, M. G. Pérez, G. M. Pérez, SeCoMan: A Semantic-Aware Policy Framework for Developing PrivacyPreserving and Context-Aware Smart Applications, IEEE Systems Journal (IF: 4.337 in 2017).

[91] M. Kolchin, D. Zamula, Food Product Ontology: Initial Implementation of a Vocabulary for Describing Food Products, in: Conference of Open Innovations Association FRUCT, 2013.

[92] M. Sabou, J. Kantorovitch, A. Nikolov, A. Tokmakoff, X. Zhou, E. Motta, Position Paper on Realizing Smart Products: Challenges for Semantic Web Technologies, in: CEUR workshop proceedings, 2009.

[93] F. Calore, D. Lombardi, E. Mussi, P. Plebani, B. Pernici, Retrieving Substitute Services Using Semantic Annotations: A Foodshop Case Study, in: International Conference on Business Process Management, Springer, 2007.

[94] V. Espín, M. V. Hurtado, M. Noguera, Nutrition for Elder Care: a Nutritional Semantic Recommender System for the Elderly, Expert Systems (IF: 1.505 in 2017).

[95] M. Boulos, A. Yassine, S. Shirmohammadi, C. Namahoot, M. Brückner, Towards an Internet of Food: Food Ontologies for the Internet of Things, MDPI Future Internet Journal.

[96] Y.-L. Chi, T.-Y. Chen, W.-T. Tsai, A Chronic Disease Dietary Consultation System Using OWL-Based Ontologies and Semantic Rules, Elsevier Journal of Biomedical Informatics (IF: 2.882 in 2017).

[97] S. Karim, U. U. Shaikh, Q. Rajput, Ontology-Based Personalized Dietary Recommendation For Travelers, in: Southern Association for Information Systems Conference (SAIS), 2015. 
[98] D. Çelik, FoodWiki: Ontology-Driven Mobile Safe Food Consumption System, The Scientific World Journal (IF: 1.219 in 2013 ).

[99] T. Pizzuti, G. Mirabelli, M. A. Sanz-Bobi, F. Goméz-Gonzaléz, Food Track \& Trace ontology for Helping the Food Traceability Control, Journal of Food Engineering.

[100] T. Pizzuti, G. Mirabelli, Ftto: An Example of Food Ontology for Traceability Purpose, in: International Conference on Intelligent Data Acquisition and Advanced Computing Systems (IDAACS), IEEE, 2013.

[101] C.-J. Su, Y.-A. Chen, C.-W. Chih, Personalized Ubiquitous Diet Plan Service based on Ontology and Web Services, International Journal of Information and Education Technology.

[102] C.-J. Su, C.-Y. Chiang, M.-C. Chih, Ontological Knowledge Engine and Health Screening Data Enabled Ubiquitous Personalized Physical Fitness (UFIT), MDPI Sensors Journal (IF: 3.031 in 2018).

[103] P. Tumnark, F. A. d. Conceição, J. P. Vilas-Boas, L. Oliveira, P. Cardoso, J. Cabral, N. Santibutr, Ontology-Based Personalized Dietary Recommendation for Weightlifting, in: International Workshop on Computer Science in Sports, Atlantis Press, 2013.

[104] R.-C. Chen, Y.-D. Lin, C.-M. Tsai, H. Jiang, Constructing a Diet Recommendation System based on Fuzzy Rules and Knapsack Method, in: International Conference on Industrial, Engineering and Other Applications of Applied Intelligent Systems, Springer, 2013.

[105] P. Curiel, A. Lago, An Infrastructure to Enable Lightweight Context-Awareness for Mobile Users, MDPI Sensors Journal (IF: 3.031 in 2018).

[106] L. Miao, J. Chun, J. C. Yoshiyuki HIGUCHI, Ontology-based User Preferences Bayesian Model for Personalized Recommendation, Journal of Computational Information Systems (IF not found).

[107] N. Suksom, M. Buranarach, Y. M. Thein, T. Supnithi, P. Netisopakul, A Knowledge-Based Framework for Development of Personalized Food Recommender System, in: International Conference on Knowledge, Information and Creativity Support Systems (Conference ranking not found), 2010.

[108] U. Yasavur, R. Amini, C. Lisetti, N. Rishe, Ontology-Based Named Entity Recognizer for Behavioral Health, in: International FLAIRS Conference, 2013

[109] G. Vadivu, S. W. Hopper, Semantic Linking and Querying of Natural Food, Chemicals and Diseases, International Journal of Computer Applications (IF: 3.12 in 2017).

[110] D. H. Fudholi, N. Maneerat, R. Varakulsiripunth, Y. Kato, Application of Protégé, SWRL and SQWRL in Fuzzy Ontology-Based Menu Recommendation, in: International Symposium on Intelligent Signal Processing and Communication Systems (Conference ranking not found), IEEE, 2009

[111] D. H. Fudholi, N. Maneerat, R. Varakulsiripunth, Ontology-Based Daily Menu Assistance System, in: International Conference on Electrical Engineering/Electronics, Computer, Telecommunications and Information Technology, IEEE, 2009.

[112] H. Gu, D. Wang, A Content-Aware Fridge based on RFID in Smart Home for Home-Healthcare, in: International Conference on Advanced Communication Technology (Conference ranking not found), IEEE, 2009.

[113] C. Snae, M. Bruckner, FOODS: a Food-Oriented Ontology-Driven System, in: International Conference on Digital Ecosystems and Technologies (DEST C-Ranked Conference), IEEE, 2008.

[114] A. Sachinopoulou, J. Leppanen, H. Kaijanranta, J. Lahteenmaki, Ontology-Based Approach for Managing Personal Health and Wellness Information, in: International Conference of the Engineering in Medicine and Biology Society, IEEE, 2007.

[115] H.-C. Li, W.-M. Ko, Automated Food Ontology Construction Mechanism for Diabetes Diet Care, in: International Conference on Machine Learning and Cybernetics (ICMLC, B-Rank Conference), IEEE, 2007.

[116] Y.-H. Chen, T.-h. Huang, D. C. Hsu, J. Y.-j. Hsu, ColorCocktail: an Ontology-Based Recommender System, 2006.

[117] R. Ribeiro, F. Batista, J. P. Pardal, N. J. Mamede, H. S. Pinto, Cooking an Ontology, in: International Conference on Artificial Intelligence: Methodology, Systems, and Applications (Conference ranking not found), Springer, 2006.

[118] J. Cantais, D. Dominguez, V. Gigante, L. Laera, V. Tamma, An Example of Food Ontology for Diabetes Control, in: Workshop on Ontology Patterns for the Semantic Web, International Semantic Web Conference (ISWC, A-Rank Conference), 2005.

[119] Open food facts project, https://world.openfoodfacts.org/who-we-are.

[120] C. Villalonga, H. Pomares, I. Rojas, O. Banos, MIMU-Wear: Ontology-Based Sensor Selection for Real-World Wearable Activity Recognition, Neurocomputing.

[121] R. Reda, A Semantic Web Approach to Ontology-based System: Integrating, Sharing and Analysing IoT Health and Fitness Data, Master's thesis (2017).

[122] E. Consoritum, Smart Body Area Networks (SmartBAN) Unified Data Representation Formats, Semantic and Open Data Model (2015).

[123] L. Nachabe, M. Girod-Genet, B. El Hassan, F. Aro, Applying Ontology to WBAN for Mobile Application in the Context of Sport Exercises, in: International Conference on Body Area Networks, Institute for Computer Sciences, Social-Informatics and Telecommunications Engineering (ICST), 2014.

[124] Y.-S. Chang, C.-T. Fan, W.-T. Lo, W.-C. Hung, S.-M. Yuan, Mobile Cloud-Based Depression Diagnosis Using an Ontology and a Bayesian Network, Elsevier Future Generation Computer Systems (IF: 4.639 in 2017)

[125] H. Jung, H.-A. Park, T.-M. Song, E. Jeon, A. R. Kim, J. Y. Lee, Development of an Adolescent Depression Ontology for Analyzing Social Data, Studies in Health Technology and Informatics.

[126] A. Rhayem, M. B. A. Mhiri, M. B. Salah, F. Gargouri, Ontology-Based System for Patient Monitoring with Connected Objects, Procedia Computer Science.

[127] J. Jokiniemi, Ontologies and Computational Methods for Traditional Chinese Medicine, Master's thesis (2010).

[128] C. Cao, Y. Sui, Building an Ontology and Knowledge Base of the Human Meridian-Collateral System, in: Innovative Techniques and Applications of Artificial Intelligence Conference, Springer, 2005.

[129] P. Budner, J. Eirich, P. A. Gloor, "Making you Happy Makes me Happy"- Measuring Individual Mood with Smartwatches, arXiv preprint.

[130] R. LiKamWa, Y. Liu, N. Lane, et al., Moodscope: Building a Mood Sensor from Smartphone Usage Patterns, in: International conference on Mobile systems, applications, and services (MobiSys - A-Rank Conference), ACM, 2013.

[131] D. Zhou, J. Luo, V. M. Silenzio, Y. Zhou, J. Hu, G. Currier, H. Kautz, Tackling Mental Health by Integrating Unobtrusive Multimodal Sensing, in: Conference on Artificial Intelligence (AAAI, A-Rank Conference), 2015. 


\begin{tabular}{|c|c|c|c|c|}
\hline Authors & Year & Project & $\mathbf{O A}$ & Reasoning \\
\hline Berthelon et al. [75] & 2013 & Emotion Ontology for Context Awareness & $\checkmark$ & $x$ \\
\hline Sanchez-Rada et al. [76] & 2013 & Onyx: Describing emotions on the web of data & $\checkmark$ & $x$ SPIN mentioned but not used \\
\hline Hastings et al. [77] & $\begin{array}{l}2012 \\
2011\end{array}$ & $\begin{array}{l}\text { MFOEM: Mental health } \\
\text { and disease ontologies }\end{array}$ & $\checkmark$ & $x$ \\
\hline Lopez et al. [78] & 2008 & Describing emotions & $\checkmark$ & $x$ \\
\hline Arguedas et al. [79] & 2015 & Emotion Awareness & $x$ & $x$ \\
\hline Tapia et al. [80] & 2014 & $\begin{array}{l}\text { Semantic Human Emotion Ontology (SHEO) } \\
\text { DetectionEmotion: Facial complex emotions }\end{array}$ & $x$ & $\begin{array}{l}\checkmark \text { SWRL rules } \\
\text { for complex emotions }\end{array}$ \\
\hline Sykora et al. [81] & 2013 & Emotive ontology & $x$ & $x$ \\
\hline Fang et al. [82] & 2010 & Relationships between colors and main meridians & $x$ & $x$ \\
\hline Grassi et al. [83] & 2009 & Human Emotions Ontology (HEO) & $x$ & $x$ \\
\hline Radulovic et al. [84] & 2009 & Smiley Ontology & $x$ & $x$ \\
\hline Benta et al. [85] & 2007 & $\begin{array}{l}\text { Context Aware Museum Guide by } \\
\text { considering users' affective state }\end{array}$ & $x$ & $\begin{array}{l}\checkmark \text { Fuzzy Logic } \\
\text { (logical inference) }\end{array}$ \\
\hline Obrenovic et al. [86] & 2005 & Emotional Cues & $x$ & $x$ \\
\hline Mathieu et al. [87] & 2005 & Ontology of Emotions and Feelings & $x$ & $x$ \\
\hline
\end{tabular}

Table 2: Ontology-based IoT emotion projects and reasoning mechanisms employed. Legend: Ontology Availability (OA)

\begin{tabular}{|l|l|l|l|l|}
\hline Authors & Year & Project & OA & Reasoning \\
\hline Peroni et al. [88] & 2016 & 20 Food Ontologies (fish, honey, etc.) & $\checkmark$ & $x$ \\
\hline Griffiths et al. [89] & 2016 & Universal Food Ontology & $\checkmark$ & $x$ \\
\hline Celdran et al. [90] & 2016 & Supermarket and location ontologies & $\checkmark$ & $\checkmark$ Semantic rules, SWRL \\
\hline Gyrard et al. [49] & 2015 & Naturopathy ontology and dataset & $\checkmark$ & $\checkmark$ Jena rule-based engine \\
\hline Kolchin et al. [91] & 2013 & Food product ontology & $\checkmark$ & $x$ \\
\hline Sabou et al. [92] & 2009 & SmartProducts: Food and recipes ontologies & $\checkmark$ & $\checkmark$ owl:Restriction rules \\
\hline Calore, Pernici et al. [93] & 2007 & Foodshop case study & $\checkmark$ & $\checkmark$ owl:Restriction rules \\
\hline Gaia & $X$ & Restaurant & $\checkmark$ & $\checkmark$ owl:Restriction rules \\
\hline Tropical Fruits & $X$ & Tropical Fruits & $\checkmark$ & $\checkmark$ owl:Restriction rules \\
\hline$X$ & $X$ & Pizza & $\checkmark$ & $\checkmark$ owl:Restriction rules \\
\hline$X$ & $X$ & Wine & $\checkmark$ & $\checkmark$ owl:Restriction rules \\
\hline Mooney & $X$ & Restaurant & $\checkmark$ & $x$ No owl:Restriction rules \\
\hline$X$ & $X$ & Beverage & $\checkmark$ & $x$ No owl:Restriction rules \\
\hline$X$ & 2000 & Beer & $\checkmark$ & $x$ \\
\hline
\end{tabular}

Table 3: Ontology-based available projects to design the integrated food knowledge base and reasoning mechanisms employed. Legend: Recommender System (RS), Ontology Availability (OA) 


\begin{tabular}{|c|c|c|c|c|}
\hline Authors & Year & Project & $\mathbf{O A}$ & Reasoning \\
\hline Espin et al. [94] & 2016 & Nutrition Diet RS for elderly people & $x$ & $\checkmark$ RS, SWRL rules, Pellet \\
\hline Boulos et al. [95] & 2015 & Survey paper: Food ontologies for IoT & $x$ & $x$ \\
\hline Chi et al. [96] & 2015 & Disease dietary consultation system & $x$ & $\checkmark$ Rule-based \\
\hline Karim et al. [97] & 2015 & Personalized Dietary RS for travelers & $x$ & $\checkmark$ RS, SWRL, Pellet \\
\hline Celik et al. [98] & 2015 & FoodWiki, Mobile safe food consumption & $x$ & $\checkmark>30$ SWRL rules, Pellet \\
\hline Pizzuti et al. [99] [100] & 2014 & Food Track \& Traceability ontology & $x$ & $\checkmark$ Pellet \\
\hline Su et al. [101] [102] & 2014 & Personalized fitness, diet plan, food & $x$ & $\checkmark$ SPIN rules, SPARQLMotion \\
\hline Tumnark et al. [103] & 2013 & Personalized dietary RS for weightlifting & $x$ & $\checkmark$ RS, SWRL, Pellet, SQWRL \\
\hline Chen et al. [104] & 2013 & Ontology-based diet RS & $x$ & $\checkmark$ RS, Jena rule, fuzzy, knapsack \\
\hline Curiel et al. [105] & 2013 & Users and Restaurant ontologies & $x$ & $\checkmark$ Jena OWL reasoner \\
\hline Miao et al. [106] & 2013 & User preferences for personalized RS & $x$ & $\checkmark$ Bayesian model, SWRL \\
\hline Suksom et al. [107] & 2013 & Personalized food RS & $x$ & $\checkmark$ RS, fuzzy inference \\
\hline Yasavur et al. [108] & 2013 & Health, food, activity, beverage & $x$ & $x$ \\
\hline Vadivu et al. [109] & 2010 & Natural food, chemicals and diseases & $x$ & $x$ \\
\hline Fudholi et al. [110] [111]] & 2009 & Menu RS & $x$ & RS, SWRL, SQWRL, fuzzy \\
\hline Gu et al. [112] & 2009 & Fridge, Food, smart home, RFID & $x$ & - Cannot access any PDF \\
\hline Snae et al. [113] & 2008 & FOODS, Food ontology & $x$ & $x$ \\
\hline Sachinopoulou et al. [114] & 2007 & Personal health and wellness ontology & $x$ & - Cannot access any PDF \\
\hline Li et al. [115] & 2007 & Food ontology for diabetes diet care & $x$ & - Cannot access any PDF \\
\hline Chen et al. [116] & 2006 & Cocktail (drink) RS and mood & $x$ & $\checkmark$ DL RACER OWL reasoner, RS \\
\hline Ribeiro et al. [117] & 2006 & Ontology for cooking & $x$ & $x$ \\
\hline Cantais et al., [118] & 2005 & Food/diet/product for diabetes control & $x$ & $\checkmark$ Ontology reasoners (Racer, Pellet) \\
\hline OpenFoodFacts [119] & - & Food products from around the world & $x$ & $x$ \\
\hline
\end{tabular}

Table 4: Non-available Ontology-based projects to design the integrated food knowledge base and reasoning mechanisms employed. Legend: Recommender System (RS), Ontology Availability (OA) 


\begin{tabular}{|c|c|c|c|c|}
\hline Authors & Year & Project & $\mathbf{O A}$ & Reasoning \\
\hline Villalonga et al. [38] [120] & 2017 & $\begin{array}{l}\text { Motivational messages for } \\
\text { physical activity coaching }\end{array}$ & $x$ & $\begin{array}{l}\checkmark \text { Pellet OWL-DL reasoner } \\
\text { SWRL }\end{array}$ \\
\hline Reda [121] & 2017 & Fitness IoT health ontology & $x$ & $\sqrt{ }$ Hermit \\
\hline $\begin{array}{l}\text { Nachabe et al. } \\
\text { [123] }\end{array}$ & $\begin{array}{l}2014 \\
2014\end{array}$ & $\begin{array}{l}\text { WBAN for mobile application } \\
\text { for sport exercises }\end{array}$ & $\checkmark$ (in PDF) [122] & $\checkmark$ SWRL, Pellet \\
\hline Estanol et al. [41] & 2017 & Obesity ontology & $\checkmark($ via email) & $\bar{x}$ \\
\hline Sogic et al. [39] & 2016 & Obesity ontology, teenagers & $x$ & $\checkmark 76$ SWRL rules, Pellet \\
\hline Kim et al. [40] & 2013 & $\begin{array}{l}\text { Obesity management ontology } \\
\text { for Android mobile application }\end{array}$ & $x$ & $x$ \\
\hline Scala et al. [42] & 2012 & Obesity ontology & $x$ & $\checkmark 40$ SWRL rules \\
\hline Huang et al. [45] & 2017 & Depression KG & $\checkmark$ (datasets) & $x$ \\
\hline Chang et al. [124] & 2015 & Depression ontology & $x$ & $\checkmark$ Bayesian networks \\
\hline Khoozani et al. [47] & 2010 & Stress Ontology (for humans) & $x$ & $x$ \\
\hline Hadzic et al. [46] & 2008 & Mental health Ontology & $x$ & $x$ \\
\hline Jung et al. [43] [125] & $\begin{array}{l}2017 \\
2015\end{array}$ & $\begin{array}{l}\text { Depression Ontology, adolescent } \\
\text { population, Twitter analysis }\end{array}$ & $x$ & $x$ \\
\hline Rhayem et al. [126] & 2017 & HealthIoT ontology & $x$ & $\begin{array}{l}\checkmark \text { Drools inference engine, } \\
7 \text { SWRL rules }\end{array}$ \\
\hline Mueller et al. [27] & 2011 & Sleep Activity Ontology & $x$ & $\checkmark$ Rules for data access \\
\hline Lin [63] & 2010 & $\begin{array}{l}\text { Ontology for meridians } \\
\text { psychatric disorder Detection }\end{array}$ & $x$ & $\checkmark$ Decision tree ML \\
\hline $\begin{array}{l}\text { Jokiniemi [127] } \\
\text { MS Thesis }\end{array}$ & 2010 & $\begin{array}{l}\text { Ontology for traditional } \\
\text { chinese medicine }\end{array}$ & $x$ & $x$ \\
\hline Cao et al. [128] & 2005 & $\begin{array}{l}\text { OMCAP: Ontology for human } \\
\text { meridian-collateral system }\end{array}$ & $x$ & $x$ \\
\hline
\end{tabular}

Table 5: Ontology-based projects for sleep, stress, fitness, obesity, and acupuncture and reasoning mechanisms employed. Legend: Ontology Availability (OA), Machine Learning (ML), Knowledge Graph (KG) 


\begin{tabular}{|c|c|c|c|c|}
\hline Authors & Year & $\begin{array}{l}\text { Research Problem } \\
\text { Addressed \& Project }\end{array}$ & $\begin{array}{l}\text { Sensor or } \\
\text { Measurement Type }\end{array}$ & Reasoning \\
\hline Nouh et al. [4] & 2019 & $\begin{array}{l}\text { Smart RS of Hybrid Learning } \\
\text { (SRHL), Well-being RS }\end{array}$ & $x$ & $\begin{array}{l}\checkmark \text { Hybrid RS, KNN } \\
\text { Unsupervised ML }\end{array}$ \\
\hline $\begin{array}{l}\text { Budner et al. } \\
{[129]}\end{array}$ & 2017 & $\begin{array}{l}\text { Detect happiness from watch } \\
\text { Correlation weather and mood } \\
\text { Correlation friends having } \\
\text { Positive or negative mood }\end{array}$ & $\begin{array}{l}\checkmark \text { Pebble smart watch } \\
\text { (to track activity), GPS } \\
\text { heart rate, light level } \\
\text { weather, humidity }\end{array}$ & $\begin{array}{l}\sqrt{ } \text { Random Forest ML } \\
\text { Weka }\end{array}$ \\
\hline Ahmed et al. [69] & 2017 & Heart attack prediction & $\begin{array}{l}\checkmark \text { Heart rate, blood pressure } \\
\text { cholesterol, blood sugar }\end{array}$ & $\checkmark$ KNN ML \\
\hline Lim [15] & 2013 & $\begin{array}{l}\text { I-Wellness: Personalized } \\
\text { wellness therapy RS }\end{array}$ & $\begin{array}{l}\checkmark \text { Health status, } \\
\text { personal lifestyle, } \\
\text { wellness concern }\end{array}$ & $\begin{array}{l}\checkmark \text { RS, Hybrid Case-Based } \\
\text { Reasoning (CBR) }\end{array}$ \\
\hline $\begin{array}{l}\text { Likamwa et al. } \\
\text { [130] }\end{array}$ & 2013 & $\begin{array}{l}\text { User's: mood mobile app } \\
\text { Correlates mood and phone usage }\end{array}$ & $\checkmark$ Phone & $\begin{array}{l}\checkmark \text { Clustering classifier } \\
\text { Multi-linear regression }\end{array}$ \\
\hline Lin et al. [16] & 2011 & $\begin{array}{l}\text { Motivate: Personalized } \\
\text { context-aware RS }\end{array}$ & $\begin{array}{l}\checkmark \text { Location, } \\
\text { weather, agenda }\end{array}$ & $\begin{array}{l}\sqrt{\text { RS, Rule-based }} \\
\text { (If-then rules) }\end{array}$ \\
\hline Lane et al. [18] & 2011 & BeWell: Well-being mobile app & $\checkmark$ Sleep, activity, social & $x$ \\
\hline Rabbi et al. [20] & 2011 & Well-being mobile application & $\begin{array}{l}\checkmark \text { Voice, accelerometer, } \\
\text { depressive symptoms }\end{array}$ & $\checkmark$ HMM \\
\hline Church et al. [21] & 2010 & $\begin{array}{l}\text { MobiMood: Mobile mood } \\
\text { awareness and communication }\end{array}$ & $\begin{array}{l}\checkmark \text { Location, calls, SMS. } \\
\text { mood intensity }\end{array}$ & $x$ \\
\hline Afzal et al. [14] & 2018 & Personalized well-being health & $\begin{array}{l}\checkmark \text { Location, activity, } \\
\text { weather, emotion }\end{array}$ & $\sqrt{ }$ RS, Rule-based \\
\hline $\begin{array}{l}\text { Garcia-Ceja et al. } \\
{[12]}\end{array}$ & 2018 & $\begin{array}{l}\text { Mental Health Monitoring } \\
\text { Systems (NHMS) Survey }\end{array}$ & $\begin{array}{l}\text { Heart rate, GSR, } \\
\text { body or skin temperature }\end{array}$ & $\begin{array}{l}\text { Survey paper } \\
\checkmark \text { ML algorithm }\end{array}$ \\
\hline Kim et al. [23] & 2017 & $\begin{array}{l}\text { Depression Severity } \\
\text { Elderly People, AAL }\end{array}$ & $\checkmark$ Infrared motion sensor & $\begin{array}{l}\text { Bayesian Network } \\
\text { Decision Tree, SVM, ANN }\end{array}$ \\
\hline Zhou et al. [131] & 2015 & $\begin{array}{l}\text { Monitoring mental health } \\
\text { states }\end{array}$ & $\begin{array}{l}\checkmark \text { Heart rate, pupil variation, } \\
\text { head movement, eye blink } \\
\text { facial expression }\end{array}$ & $\begin{array}{l}\checkmark \text { ML (Logistic } \\
\text { regression, SVM) }\end{array}$ \\
\hline $\begin{array}{l}\text { Garcia-Ceja et al. } \\
{[24]}\end{array}$ & $\overline{2016}$ & "Stress & $\begin{array}{l}\text { ( Accelerometer data } \\
\text { (from smartphone) }\end{array}$ & $\begin{array}{l}\checkmark \text { Naive Bayes, } \\
\text { Decision Tree }\end{array}$ \\
\hline Yoon et al. [33] & 2016 & New stress monitoring patch & $\begin{array}{l}\checkmark \text { Skin conductance, } \\
\text { pulse wave, skin temperature }\end{array}$ & $x$ \\
\hline Lu et al. [25] & 2012 & StressSense & $\checkmark$ Voice data (smartphone) & $\checkmark$ GMMs \\
\hline Chang et al. [26] & 2011 & AMMON: Stress detector & $\checkmark$ Voice data & $\checkmark \mathrm{SVM}$ \\
\hline $\begin{array}{l}\text { Yacchirema et al. } \\
\text { [30] }\end{array}$ & 2018 & Obtrusive Sleep Apnea (OSA) & $\begin{array}{l}\text { Heart rate, snoring, activity, } \\
\checkmark \text { BMI, weight, step counts } \\
\text { temperature, humidity } \\
\text { air pollutant }\end{array}$ & $\begin{array}{l}\checkmark \text { Rule-based } \\
\text { ANN-MLP RELU }\end{array}$ \\
\hline Angelidou [29] & 2015 & Sleep apnea diagnosis, snoring & $\sqrt{ }$ Bio-signal and sound data & $\sqrt{ }$ Decision tree $($ Weka) \\
\hline $\begin{array}{l}\text { Laxminarayan } \\
{[28]}\end{array}$ & 2004 & Exploratory sleep analysis & $\begin{array}{l}\checkmark \text { Heart rate, } \\
\text { oxygen potential, body position }\end{array}$ & $\begin{array}{l}\checkmark \text { Association rule mining } \\
\text { Logistic regression, Weka }\end{array}$ \\
\hline
\end{tabular}

Table 6: Well-being and IoT-based emotion applications (positive and negative). Sensor measurement and reasoning taxonomy to later process sensor data with reasoning mechanisms within applications. Set of keywords relevant for automatic analysis. Legend: Machine Learning (ML), Support Vector Machine (SVM), Recommender System (RS), Ambient Assisted Living (AAL), Gaussian Mixture Models (GMMs), Artificial Neural Network (ANN), Multilayer Perceptron (MLP), Galvanic Skin Response (GSR), Hidden Markov Model (HMM), K-Nearest Neighbors (KNN) 